Gabriel and Heddle, Proc. roy. Soc. A, 258, 124, 1960 (Ea; $n$ SPD - $m$ SPD).-St. John, Bronco and Fowler, Y. opt. Soc. Amer. 50, 28, I $960\left(E a ;\right.$ I $\left.S-3{ }^{1} P\right)$.

$\mathrm{He}^{+} \quad$ Burgess, M.N. 132, 503, 1960 .

Ar Volkova and Devyatov, Optika i Spektr. 7, 819, 1959 (a).-Volkova, Bull. Acad. Sci. U.R.S.S. Ser. Phys. 23, 968, 1959 (Ea).

K Volkova, Bull. Acad.Sci.U.R.S.S. Ser. Phys. 23, 968, 959 (Ea); Optika i Spektr. 6, 179, $1959(E a ; 6 P-4 S)$.

$\mathrm{Ca}^{+} \quad$ van Regemorter, M.N. I21, 213, I960 $(T a ; 4 s-4 p, 3 d-4 p)$.

$\mathrm{Hg} \quad$ Rozgachev, Optika i Spektr. 4, 549, $195^{8}\left(E a ; 6^{1} S-6^{3} P, 7^{3} S\right)$.

Fe XIv Blaha, Bull. Astr. Inst. Czech. 9, 160, 1958 (Ta).-Schwarz and Zirin, Ap. F. 130, 384, I959 (Ta).-Burgess, $A p$. F. 132, 503, 1960.

$\mathrm{N}_{2} \quad$ Stewart and Garbathuler, Proc. phys. Soc. London 72, 287, 1958 $\left(E a ; C^{3} \Pi-B^{3} \Pi\right)$. Kishko and Kuchinka, Optika $i$ Spektr. 6, 378, I959 (Er; $\left.C^{3} \Pi-B^{3} \Pi\right)$.- Schulz, Phys. Rev. I16, I141, 1959.

CO Schulz, Phys. Rev. 116, I 141, 1959.

$\mathrm{O}_{2}{ }^{+} \quad$ Stewart and Garbathuler, Proc. phys. Soc. London 72, 287, $1958\left(E a ;{ }^{+} \Sigma_{\mathrm{g}}-{ }^{4} \Pi_{\mathrm{u}}\right.$ and $\left.A^{2} \Pi_{u}-X^{2} \Pi_{g}\right)$.

$\mathrm{N}_{2}{ }^{+} \quad$ Zapesochny and Kishko, Bull. Acad. Sci. U.R.S.S. Ser. Phys. 23, $965,1959(E)$.

General paper:

Milford, $A p . \mathcal{F}$. 131, 407, 1960 .

\title{
14b. SOUS-COMMISSION DES SPECTRES MOLECULAIRES D'INTERET ASTRONOMIQUE
}

Prísident : Professeur P. Swings, Directeur de l'Institut d'Astrophysique de l'Université de Liège, Cointe-Sclessin, Liège, Belgium.

Membres: Herzberg, Junkes, McKellar†, Mme Moore-Sitterly, Nevin, Phillips.

EN PIEUX HOMMAgE À LA MÉMOIRE DE FRANCIS A. JENKINS

ET ANDREW MCKELLAR

Les besoins des astro-spectroscopistes en informations sur les spectres de bandes ont certes continué à se manifester dans le domaine spectral usuel d'observation, approximativement de $\lambda_{3000}$ à $\lambda 9000 \AA$. Mais il faut, à présent, penser aussi aux besoins en spectres moléculaires dans le domaine astronomique ultra-violet lointain qui commence à s'ouvrir à nous grâce aux observations à bord des fusées et satellites. On ne peut, non plus, négliger le domaine astronomique infra-rouge qui commence à être accessible, avec une résolution assez élevée, soit grâce aux nouvelles émulsions photographiques, soit par l'emploi des récepteurs infra-rouges de plus en plus sensibles, combinés aux systèmes dispersifs de principe nouveau récemment développés (par exemple l'interféromètre de Michelson avec transformée de Fourier). Dans l'infra-rouge astronomique, les spectres moléculaires joueront sûrement un rôle considérable.

Le spectre ultra-violet lointain de $\mathrm{H}_{2}$ qui, jusqu'ici, n'avait pas présenté un intérêt astronomique très marqué, revêtira vraisemblablement bientôt une importance considérable, car $\mathrm{H}_{2}$ est, sans doute, la molécule la plus abondante dans l'atmosphère de nombreux astres et, peutêtre aussi, dans l'espace interstellaire et interplanétaire. Il est donc heureux qu'une nouvelle étude soigneuse du spectre de $\mathrm{H}_{2}$ soit actuellement en cours (I) (2) (3) (4). 
Nous pouvons nous attendre à ce que, dans un avenir rapproché, de nouvelles pénuries graves en informations spectroscopiques moleculaires se manifestent dans les domaines ultraviolet et infra-rouge.

Je ne discuterai cependant pas ici ces besoins nouveaux pour lesquels je renvoie au rapport de la Commission 44. Mais, même dans la région spectrale usuelle, la coopération étroite des spectroscopistes moléculaires est encore bien nécessaire aux astro-spectroscopistes. Madame C. Moore Sitterly l'a montré clairement dans le cas des identifications solaires. La présence d'une vingtaine de molécules a été suggérée dans le soleil, mais certaines identifications sont plutôt douteuses. Au cours de l'actuel triennat, Madame Sitterly (5) (qui avait examiné CH, CN et $\mathrm{OH}$ en détail antérieurement) a étudié les identifications solaires de $\mathrm{C}_{2}, \mathrm{NH}$ et $\mathrm{MgH}$ et elle s'occupe actuellement de $\mathrm{SiH}$. Environ $45^{\circ}$ raies de $\mathrm{C}_{2}$ et 256 de $\mathrm{NH}$ sont présentes, dont respectivement $18 \%$ et $35 \%$ sont 'blended'. Les raies de $\mathrm{MgH}$ sont faibles dans le spectre du disque, mais fortes dans celui des taches; 96 raies de $\mathrm{MgH}$ (dont un tiers sont blended) ont été identifiées dans le spectre du disque. Les molécules restantes devraient être étudiées en détail dans le spectre des taches. Il est, d'ailleurs, certain que les identifications solaires pourront être considérablement étendues, lorsque des données de laboratoire plus précises et plus détaillées seront disponibles; bien entendu, c'est surtout dans le spectre des taches que ces identifications moléculaires seront les plus nombreuses. Le bel Atlas Molecular Spectra of Metallic Oxides préparé par notre regretté collègue, le Père A. Gatterer, et ses collaborateurs, les Pères J. Junkes et E. W. Salpeter avec la coopération de B. Rosen se révèlera de plus en plus utile dans de telles études.

Les besoins des astronomes sont bien connus de certains éminents spectroscopistes de laboratoire, dont l'aide nous est fort précieuse. Nous devons rendre un hommage particulier à l'entreprise commencée en février 1959, au laboratoire de spectroscopie moléculaire de l'Université de Californie à Berkeley. A l'origine de cet effort, se trouvaient F. A. Jenkins (du Département de Physique) et J. G. Phillips (du Département d'Astronomie). Hélas, nous avons récemment perdu notre savant collègue et ami, F. A. Jenkins. Son associé, J. G. Phillips, a décidé de continuer en collaboration avec S. P. Davis, du Département de Physique, l'oeuvre importante à laquelle Jenkins s'était attaché, en utilisant le grand réseau et les ressources du Bureau de Calculs de Berkeley dont une IBM 704 (6). Les mesures, réductions et analyses sont effectuées de façon efficace et rapide; les résultats seront publiés suivant le même modèle que les tables moléculaires de Madame Sitterly. Après leur analyse des bandes du système rouge de $\mathrm{CN}$, Phillips et Davis comptent entreprendre l'étude et la publication des analyses des bandes de $\mathrm{C}_{2}, \mathrm{NH}, \mathrm{BH}, \mathrm{MgH}, \mathrm{AlH}, \mathrm{SiH}, \mathrm{SiF}, \mathrm{BO}, \mathrm{TiO}, \mathrm{ZrO}, \mathrm{AlO}, \mathrm{ScO}, \mathrm{LaO}$ et YO; cette liste de molécules est conforme aux voeux de Madame Sitterly et des autres astrospectroscopistes.

Parmi les laboratoires actifs dans la spectroscopie des molécules d'intérêt astronomique, celui d'Ottawa a continué à se distinguer particulièrement. Outre des études sur $\mathrm{H}_{2}$ et $\mathrm{HD}$ (r) (2) (3) on y a effectué des recherches sur les hydrures $\mathrm{CH}^{+}(7)$ (d'ou a résulté l'identification de la dernière raie interstellaire fine inexpliquée, $\lambda_{3579}$ ), $\mathrm{NH}(8), \mathrm{PH}(9)$, et $\mathrm{CrH}$ (ro); sur $\mathrm{C}_{2}$ (II) (pour laquelle il a été montré que l'état normal est ${ }^{1} \Sigma_{g}^{+}$et non ${ }^{3} \Pi_{u}$ ); sur $\mathrm{N}_{2}^{+}$(systèmes $\mathrm{A}-\mathrm{X}(\mathbf{1 2})$ et $\mathrm{C}-\mathrm{X}(\mathbf{1 3})$; bande nouvelle (14)), $\mathrm{O}_{2}$ (15) (prédissociation dans les bandes de Schumann-Runge), $\mathrm{P}_{2}(\mathbf{1 6})$ (nouveau système près de $\lambda_{3000}$ ) et les oxydes $\mathrm{PO}\left(\mathbf{I}_{7}\right), \mathrm{ClO}$, $\mathrm{BrO}$ et $\mathrm{IO}(\mathbf{r 8})$. On a aussi étudié, en absorption, diverses molécules polyatomiques simples: $\mathrm{HCN}$ et DCN (19), $\mathrm{NH}_{2}(\mathbf{2 0})$; $\mathrm{HNO}(\mathbf{2 1}), \mathrm{NCO}(\mathbf{2 2}), \mathrm{CH}_{2}$ (23) et $\mathrm{CH}_{3}$ (24).

Parmi les autres travaux, signalons ceux du laboratoire de Professor T. E. Nevin à Dublin (nouveaux systèmes infra-rouge et vert Gaydon-Herman de $\mathrm{N}_{2}$ par $\mathrm{P}$. K. Carroll et collaborateurs (13) (14) (r5) (25); bandes de OD ${ }^{+}$par S. O'Connor (26), de la Specola Vaticana (bandes 
de HfO par J. Junkes (27)), de Moscou (probabilités de transition du système SchumannRunge de $\mathrm{O}_{2}(\mathbf{2 8})$ ), de Bâle (travaux de E. Miescher sur NO (29)), de Stockholm (étude d'oxydes divers, $\mathrm{BO}\left(\mathbf{3}^{\circ}\right)$, $\mathrm{NO}$ (3r), $\mathrm{AlO}\left(\mathbf{3 2}^{2}\right), \mathrm{LaO}(33)$, par le groupe de A. Lagerqvist), de Chicago (P. G. Wilkinson, $\mathrm{N}_{2}(34)$ ), d'Oxford (R. F. Barrow, BF (35), GeF (36), $\mathrm{HCl}$ (37), $\mathrm{HF}^{+}\left(3^{8}\right), \operatorname{SiF}(39) \ldots$ ), de Baltimore (G. H. Dieke, $\mathrm{H}_{2}\left(\mathbf{4 0}^{0}\right)$, de Cambridge (Y. Tanaka et collaborateurs, $\mathrm{N}_{2}(4 \mathrm{I}), \mathrm{CO}_{2}(42), \mathrm{N}_{2} \mathrm{O}(42), \mathrm{He}_{2}$ (43), de Liège (B. Rosen, $\mathrm{CaO}$ (44), LaO (45), $\mathrm{TiO}\left(4^{6}\right), \mathrm{ZrO}\left(4^{6}\right)$, $\mathrm{ThO}\left(4^{6}\right)$, $\mathrm{VO}(47), \mathrm{CrO}(47)$, oxydes de terres rares (47), etc. . .

Il faut aussi signaler la compilation des calculs théoriques ("Basis Functions for $\mathrm{Ab}$ Initio Calculations') de L. C. Allen et A. M. Karo (48), relative aux fonctions propres moléculaires. Des résultats théoriques existent pour les molécules d'intérêt astronomique $\mathrm{H}_{2}, \mathrm{He}-\mathrm{He}$, $\mathrm{H}_{2}+\mathrm{H}_{2}, \mathrm{H}_{4}, \mathrm{LiH}, \mathrm{BeH}^{+}, \mathrm{BEH}, \mathrm{BH}, \mathrm{CH}^{+}, \mathrm{CH}, \mathrm{NH}, \mathrm{OH}, \mathrm{NH}_{2}, \mathrm{CH}_{3}, \mathrm{HF}, \mathrm{H}_{2} \mathrm{O}, \mathrm{NH}_{3}$, $\mathrm{NH}_{4}^{+}, \mathrm{CH}_{4}, \mathrm{C}_{2}, \mathrm{LiF}, \mathrm{N}_{2}, \mathrm{BF}, \mathrm{CO}, \mathrm{HCN}, \mathrm{NO}, \mathrm{O}_{2}, \mathrm{CO}_{2}$ et $\mathrm{O}_{3}$.

Conformément à l'intitulé de la Sous-Commission, nous ne discuterons ici ni la question des intensités, ni les observations astronomiques dans lesquelles interviennent les spectres moléculaires. Ces problèmes seront, sans doute, traités dans d'autres rapports.

\section{P. SWINGS}

Président de la Sous-Commission

\section{RÉFÉRENCES}

I. Herzberg, G. and Howe, L. L.

Canad. F. Phys. 37, 636, 1959.

2. Durie, R. A. and Herzberg, G.

Canad. F. Phys. 38, 806, 1960.

3. Herzberg, G. and Monfils, A. F. Mol. Spectr. à l'impression (1960).

4. Monfils, A. Communication privée (décembre I960).

5. Moore Sitterly, C. Communication privée (octobre I960).

6. Phillips, J. G. Ap. F. 130, 308, I 959 (description du programme initial, utilisant une calculatrice IBM 701). Pour un exposé du nouveau programme utilisant une IBM 704. voir rapport de J. G. Phillips et S. P. Davis à la réunion d'Ottawa de la Commission Triple de Spectroscopie, 1960 .

7. Douglas, A. E. and Morton, J. R. Ap. F., 131, 1, 1960.

8. Dixon, R. N. Canad. F. Phys. 37, 1 I 7 I, 1959.

9. Legay, F. Canad 7 . Phys. 38, 797 I96o.

10. Kleman, B. and Uhler, U. Canad. F. Phys. 37, 537, 1959.

I1. Ballik, E. A. and Ramsay, D. A. F. chem. Phys. 29, 1418, 1958; 31, I128, 1959.

12. Liu, I. D. $\quad A p . \mathcal{F} .129,516$, 1959.

13. Carroll, P. K. Canad. F. Phys. 37, 880, r959.

14. Carroll, P. K. Canad. F. Phys. 36, 1585, 1958.

15. Carroll, P. K. Ap. F. 129, 794, 1959.

16. Douglas, A. E. and Rao, K. S. Canad. F. Phys. 36, 565, 1958.

17. Rao, K. S. Canad. F. Phys. 36, 1526, 1958.

18. Durie, R. A. and Ramsay, D. A. Canad. F. Phys. 36, 35, 1958; Durie, R. A., Legay, F. and Ramsay, D. A. Canad. Y. Phys. 38, 444, 1960.

19. Herzberg, G. and Innes, K. K. Canad. F. Phys. 35, 842, 1957.

20. Dressler, K. and Ramsay, D. A. Phil. Trans. 25rA, 553, r959.

2r. Dalby, F. W. Canad. F. Phys. 36, 1336, 1958.

22. Dixon, R. N. Canad. F. Phys. 38, 10, 1960; Phil. Trans. 252A, 165, 1960.

23. Herzberg, G. and Shoosmith, J. Nature, Lond. 183, I80r, I959.

24. Herzberg, G. (en préparation).

25. Carroll, P. K. and Rubalcava. H. E. Nature, Lond. 184, I I9, 959.

26. Nevin, T. E. Communication privée (octobre I960).

27. Junkes, J. Communication privée (octobre 1960).

28. Biberman, L. M., Erkovich, S. P. et Sashnikov, V. N. Optika i Spektr., 7, 562, I959. 
29. Miescher, E. Communication au ro ème Colloque Intern. Astroph. Liège, 1960; Lagerqvist, A. and Miescher, E. Helv. Phys. Acta, 31, 221, 1958.

30. Lagerqvist, A., Nilsson, N. E. L. and Wigartz, K. Ark. Fys. 13, 379, 1958.

3r. Lagerqvist, $\mathrm{A}$. and Miescher, $\mathrm{E}$. loc. cit.

32. Lagerqvist, A. Nilsson, N. E. L. and Barrow, R. F. Ark. Fys. 12, 543, 1957.

33. Lagerqvist, A. and Huldt, E. Ark. Fys. 12, 491, 1957.

34. Wilkinson, P. G. F. chem. Phys. 30, 773, 1959.

35. Barrow, R. F. Premaswarup, D., Winternitz, J. and Zeeman, P. B. Proc. phys. Soc. Lond. 7IA, 6I, 1958.

36. Barrow, R. F., Butler, D., Johns, J. W. C. and Powell, J. L. Proc. phys. Soc. Lond. 73A, $317,1959$.

37. Jacques, J. K. and Barrow, R. F. Proc. phys. Soc. Lond. 73A, 538, 1959.

38. Johns, J. W. C. and Barrow, R. F. Nature, Lond. 179, 374, 1957.

39. Johns, J. W. C. and Barrow, R. F. Proc. phys. Soc. Lond. 7XA, 476, 1958.

40. Dieke, G. H. F. Mol. Spectr. 2, 494, 1958.

4I. Ogawa, M. and Tanaka, Y. F. chem. Phys. 30, r354, I959; Leblanc, F., Tanaka, Y. and Jursa, A. F. chem. Phys. 28, 979, 1958.

42. Tanaka, Y. Jursa, A. S. and Leblanc, F. J. F. chem. Phys. 28, 350L, 1958.

43. Tanaka, Y., Jursa, A. S. and Leblanc, F. J. F. opt. Soc. Am. 48, 304, 1958.

44. Rosen, B. et Weniger, S. C.R. 248, I645, 1959; F. mol. Spectr., à l'impression 7, 196r.

45. Rosen, B. et Hautecler, S. Bull. Ac. R. Belg., Cl. Sc. 95, 790, 1959.

46. Rosen, B. F. mol. Spectr., à l'impression 7, 196r.

47. Rosen, B. Communication privée (décembre 1960 ).

48. Allen, L. C. and Karo, A. M. Rev. mod. Phys. 32, 275, 1960.

Addendum: Alors que le présent rapport était terminé, j'ai recu la Bibliography of Spectra of Diatomic Molecules, 1950-1960 par G. Herzberg, and L. L. Howe. On y trouvera toutes les autres références relatives aux travaux spectroscopiques récents sur les molécules diatomiques.

J'ai aussi reçu de Madame $R$. Herman, les reférences suivantes:

49. Herman, L., Ferguson, H. I. S. and Nicholls, R. W. Canad. F. Phys. 39, 476, 196r $\left(\mathrm{O}_{2}^{+}\right)$.

50. Herman, L. et Rakotoarinimy, D. Fournal de Physique et le Radium, 2x, 629, r960 (CO et $\mathrm{N}_{2}$ ).

51. Herman, L., Felenbok, P. et Mme Herman, R. Journal de Physique et le Radium, 22, 83, I96I (OH et OD).

52. Herman, L., Mme Herman, R. et Rakotoarinimy, D. Fournal de Physique et le Radium, 22, $\mathrm{I}, \mathrm{Ig6r}\left(\mathrm{O}_{2}\right)$. 